\title{
Systematization of Nursing Assistance: Perspectives of the Applicability in the Care of the Person with Diabetes and Hypertension
}

\author{
Desirée Costa Bezerra ${ }^{1}$, Cecilia Rafaela Salles Ferreira ${ }^{2}$, Francineide Pereira da Silva Pena ${ }^{3}$, \\ Crislayne Simões Pereira ${ }^{1}$, Diurieny Ribeiro Itaparica Vieitas ${ }^{1}$, José Luís da Cunha Pena ${ }^{3}$, \\ Ananda Larisse Bezerra da Silva ${ }^{2}$
}

${ }^{1}$ Collective Health, Macapá, Brazil

${ }^{2}$ Basic Health Unit of Federal University of Amapá, Macapá, Brazil

${ }^{3}$ Nursing Collegiate of Federal University of Amapá, Macapá, Brazi

Email address:

desicb2393@gmail.com (D. C. Bezerra)

\section{To cite this article:}

Desirée Costa Bezerra, Cecilia Rafaela Salles Ferreira, Francineide Pereira da Silva Pena, Crislayne Simões Pereira, Diurieny Ribeiro Itaparica Vieitas, José Luís da Cunha Pena, Ananda Larissa Bezerra da Silva. Systematization of Nursing Assistance: Perspectives of the Applicability in the Care of the Person with Diabetes and Hypertension. American Journal of Nursing Science.

Vol. 8, No. 4, 2019, pp. 174-180. doi: 10.11648/j.ajns.20190804.19

Received: May 2, 2019; Accepted: June 18, 2019; Published: July 8, 2019

\begin{abstract}
The systematization of nursing care, is a action private of nurses, ensuring better planning of nursing actions and processes. Thus, the study has the objective indetify nurses' perspectives and updates on the application of systematization of nursing care for people with diabetes mellitus and systemic arterial hypertension. It's a qualitative study, of the type practical case of action, carried out at the Basic Health Unit of the Federal University of Amapá, in 2018, with participation of six nurses. The data collection for the interview and a focus group, analysis of results in light of the theory of studies related to the subject. Three themes were identified: Potentials and perspectives in the application of the Systematization of Nursing Assistance in Primary Care; Expected difficulties and difficulties encountered; The use of the Systematization of Nursing Assistance in Primary Care: before and after a practical experience. The implementation of the systematization of care still presents difficulties, however, in the medium term, it will bring more benefits than any burden identified for the assistance and management of human resources and materials of the health unit, promoting interventions more resoluteness and a planning of actions based in scientific knowledge, bringing legal and technical support for the professionals nurses and all team involved.
\end{abstract}

Keywords: Nursing Care, Primary Nursing Care, Nursing Consultation, Nursing Process, Chronic Disease

\section{Introduction}

The Systematization of Nursing Assistance (SNA) is a private method of nursing, which reinforces the scientific character of the profession, which guides the planning, intervention and evaluation of the nursing process, providing subsidies for solving situations of fragility in the health/disease process [1].

Thus, as an organizational process, the development of SAE should be predicted in all segments of nurses' performance. Non-communicable Chronic Diseases (NCD) care requires a multidisciplinary quality of care, nurses must be aware of their professional practice with the implementation of the use of SAE for their work to be recognized and valued, there are several strategies of care to deal with the chronic condition, which are important parts of the care, the standardization of this care occurs through the SAE [2].

Characterized and described as a chronic condition, DCNTs (Diabetes, cardiovascular diseases and others) are persistent health conditions. They require proactive social responses, which in Brazil and in the world are the result of the rapid demographic and epidemiological transition of aging populations, accompanied by risk factors for sedentary lifestyle, obesity and smoking, which has been challenging the health system in its control [3]. 
Systemic Arterial Hypertension (SAH) and Diabetes Mellitus (DM) are DCNTs with higher population occurrence and clinical conditions of a multifactorial nature. SAH is characterized by elevated and sustained blood pressure (BP), associated with changes or lesions in target organs (heart, brain, kidneys and blood vessels) [4], and DM is a heterogeneous group of metabolic disorders that shows signs of hyperglycemia as a result of defective secretion and/or insulin action [5].

Because they are chronic conditions, hypertension and DM, they present evolutionary characteristics that evidence the need for follow-up in Primary Care (PA) for management and control of the disease. Actions and practices of education, health promotion, and disease / injury prevention are essential characteristics that the nurse should have in caring for the person with CNCDs. The growth of chronic conditions attracts a new focus of attention and care to the PHC, with the need for a nursing prepared for this need for continuous and specialized service, which has interfaces, individuality and specific needs for each care [3-6].

In this context, chronic diseases, responsible for a growth in premature mortality in Brazil, which is considered a public health problem in the country [6], requires a social response from all sectors of society, being the responsibility of nursing development technologies and the use of SAE in the care to guarantee a quality assistance.

The National Basic Attention Policy (Politica Nacional de Atenção Básica - PNAB), envisaged in Decree No. $2,436 / 2017$, calls for integral attention to people in order to generate an impact on their health and autonomy, as well as on the social determinants present in the community, that is, promote individual and collective intervention strategies [7].

During the performance as a nursing resident in collective health in a Basic Health Unit, it was observed that despite the nursing consultation for people with DM and SAH, the SNA was not used as a follow-up tool for these people. Taking into account that Nursing consultations make it possible to follow the health situation, the development of a link between professional/user, the feasibility of applying SNA, as a relevance for those who will use it, the possibility of providing autonomy for both the user and for the nurse who could have a better planning of their care and that is what this study proposed.

The use of SNA as a nurse's work method allows the planning of individual and collective interventions as a strategy geared to the needs of each person. Failure to use the method results in ineffective results and dissatisfaction with the care provided, and may promote non-adherence to treatment. In view of the above, the research questions emerged: What are the perspectives of nurses in primary care regarding the applicability of SNA in nursing consultation to people with DM and SAH? What are the main difficulties identified in the applicability of SNA?

The research aimed to identify the perspectives and difficulties of nurses on the applicability of SNA in the PC to the person with DM and SAH.

\section{Method}

It consisted of a qualitative study of the type "researchaction", using as method the focus group. The study was conducted at the Basic Health Unit of the Federal University of Amapá (UNIFAP), Macapá / Amapá in the year 2018. The unit covers several Primary Care programs determined by the Ministry of Health with multidisciplinary care, among them the Program of Chronical Diseases that works only in the unit, and the Health Promotion Program for the Person with Diabetes, an extension program that provides a service that provides in addition to consultations, physical activity, continued health education, and multidisciplinary care.

The study population were nurses $(n=6)$, whose inclusion criteria were nurses who became available to participate in the study, who performed nursing consultations with people with SAH and DM in the morning and afternoon shifts, and signed the Free Consent Term and Clarified (FCTC).

Data collection took place in two stages: Initially a semistructured interview was conducted face to face with open questions about the nurses' perspective on the SNA. The second group was the focal group, which began with a handout the participants' statements regarding the interview responses. After presenting the clothesline, the nurses were invited to re-read, observing the identification with one of the lines. Following, the same interview question was repeated, the purpose of which was to observe whether the statements of the first stage were affirmed, or whether there was influence in the focus group on the perspective of each subject, since there was discussion among the participants on the topic.

The analysis of this moment resulted in the perspectives and difficulties of the nurses for the implantation of SNA. The BHU, because it was inserted in an academic context, since already existed four previous studies that listed the main nursing diagnoses for people with DM and/or with $\mathrm{SAH}$, carried out in the Program of Health Promotion mentioned above, and one of the studies investigated the nursing diagnoses in people with hypertension. From these studies, 33 diagnoses were identified and a guideline manual containing prescriptions related to diagnoses was prepared. This subsidized the implementation of the SNA.

Subsequently, during the use of the manual in the consultations, a new semistructured interview was conducted, whose purpose was to evaluate the application of SNA in the follow-up of people with SAH and DM.

The interview instrument included questions about the professional profile of the participating nurse, as well as the training time, length of service and weekly workload. In the interview of the first stage, the questions were addressed to the perspective and difficulties of the nurse about the applicability of SNA whose guiding questions were: What perspectives of nurses in the primary care as the applicability of SNA in the nursing consultation to people with DM and SAH? What are the main difficulties identified in the applicability of SNA?

The interviews and the focus group meeting were recorded 
with permission from the participants and transcribed into the Microsoft Word application for content analysis.

The analysis of the data was performed through content analysis, using as the technique the thematic analysis. Content analysis is about research techniques that allow the description of the messages and attitudes related to the context of the enunciation, in a systematic way, as well as the interferences on the collected data [8], "indicators (quantitative or not) that allow the inference of knowledge regarding the conditions of production/reception of these messages" [9].

Thus, the categories were identified from coding units, resulting from the similarity in the participants' discourse, individual interview products and the focal group meeting, before and after the SNA implementation in the service. Three categories were identified: Potentials and perspectives in the application of SNA in PC; Expected difficulties and difficulties encountered and Use of SNA in PC: before and after practical experience.

The names of the participants were replaced by codes (E1, E2, E3...) to ensure the preservation of anonymity. The results were qualitatively evaluated in a descriptive way, where the participants' responses illustrated the results.
The research complied with the precepts of Resolution No. 466/2012, of the National Health Council (Conselho Nacional de Saude - CNS), which deals with studies with human beings and was evaluated by the Research Ethics Committee (Comitê de Ética em Pesquisa - CEP) of the Federal University of Amapá, approved with an opinion No. 2.932.911, CAAE: 95598418.2.0000.0003.

\section{Results}

Of the six nurses interviewed, $2(33 \%)$ were male and 4 (67\%) female, ages, $5(83 \%)$ were up to 35 years and 1 $(17 \%)$ over 55 years. Interviewees $(100 \%)$ have a weekly workload of 40 hours, $3(50 \%)$ have more than one employment relationship. $4(67 \%)$ have up to 5 years of studies, $1(17 \%)$ is 10 years and 32 years respectively.

Table 1 describes the categories and their respective coding units resulting from the interview and the focus group before and after the application of SNA in the care of the person with DM or SAH. It is observed that the categories remained for the two moments, presenting indicators that resemble and contrast.

Table 1. Characterization of the categories in the SNA application.

\begin{tabular}{|c|c|c|c|c|}
\hline CATEGORIES & INDICATORS (BEFORE) & $(\mathbf{N}) \%$ & INDICATORS (AFTER) & $(\mathbf{N}) \%$ \\
\hline \multirow{7}{*}{$\begin{array}{l}\text { Potentials and Perspectives in } \\
\text { the Application of SNA in the } \\
\text { PC }\end{array}$} & Organization and Planning & $(5) 83 \%$ & Positive Perspectives on the Use of SNA & $(3) 75 \%$ \\
\hline & Autonomy & (2)33\% & Possibility to keep in routine & (4) $100 \%$ \\
\hline & Scientific knowledge & (1) $17 \%$ & Possibility of organizing the demands of the Service & (4) $100 \%$ \\
\hline & Scientific knowledge & (1) $17 \%$ & Better Adherence (Continuity of Care) & (2) $50 \%$ \\
\hline & Quality and objectivity in service & (2) $33 \%$ & Enhancement of technology (application) & (1) $25 \%$ \\
\hline & Better adherence to treatment & (2)33\% & & \\
\hline & Low cost technology & (1) $17 \%$ & & \\
\hline \multirow{3}{*}{$\begin{array}{l}\text { Difficulties expected, } \\
\text { difficulties found }\end{array}$} & Work overload & (2)33\% & Longer time in consultations & (4) $100 \%$ \\
\hline & Team work & (2)33\% & & \\
\hline & $\begin{array}{l}\text { Disinformation about the nursing } \\
\text { consultation for people with SAH/DM }\end{array}$ & (3) $50 \%$ & & \\
\hline \multirow{3}{*}{$\begin{array}{l}\text { Use of SNA in PC: before and } \\
\text { after practical experience }\end{array}$} & Lack of computerization & $(1) 17 \%$ & & \\
\hline & Non-systematized service & (6) $100 \%$ & Improved Quality of Consultation & (3) $75 \%$ \\
\hline & Lack of objectivity & (1) $17 \%$ & Applying existing knowledge & (1) $25 \%$ \\
\hline
\end{tabular}

\section{Discussion}

\subsection{Potentialities and Perspectives in the Application of Sna in pc}

This category evidences the positive perspective observed for the applicability of the SNA in the service, either before or after the SNA application.

In the initial interview, $83 \%$ of the interviewed nurses consider systematization as a method of organization and planning, and according to $33 \%$ of the interviewees, provides autonomy to the nurse.

The SNA guides the planning and organization of nursing actions and the team involved in this process, enabling improvement, efficiency and validity in nursing care [10], which is evidenced in the following statements:

It is through SNA that we can organize our service (E1)
It is used to strengthen the role of nurses in care (E2)

So I see that in basic care, nurses have more autonomy to develop SNA. (E3)

Still considering the methodology, 1 (17\%) considered that the use of SNA gives a scientific character to the profession, and emphasized in their speech that the basic health unit in question has a particularity: Being inserted in an academic environment, which can facilitate the implementation of SNA due to the constant flow of teachers and academics in the environment.

The planning of nursing actions permeate the establishment of goals and expected results [10].

As part of a multidisciplinary team, developing specific skills and competencies of the nurse, SAE is an important practical tool essential to care, its use demonstrates commitment to the care provided, raising the scientific and professional performance of nursing [11].

SNA is that it is step by step, the procedure that determines 
the step by step of nursing care, so it gives the scientific character. (E6)

We are part of a university. I think it would be easier for us. (E1)

The SNA was quoted by $33 \%$ of the sample as an instrument that confers quality and objectivity in the service, since it allows an individualized service and directed to the main demands evidenced by the client, also guarantees the continuity of care, because through the evaluation is checked the effectiveness of planning and assess new demands, improving adherence to treatment. This speech is evidenced in the sample.

For example, we knowing how to identify the serious demands of that patient, so as to guide and lead people in the SNA process, knowing how to identify in a way that does not prolong the consultation. (E1)

I think this issue of follow-up is primary because with the instrument in the patient's chart we can see what has already been done and are looking for it from the patient, whether he did or did not, and check other things that were not noticed before. (E4)

These statements corroborate the SAE definition by the references [10-12] through systematization, it is possible to organize the work on the method, it provides visibility, and ensures the scientificity to the nurses' performance, providing autonomy to the nurse and patient safety.

The methodology was considered in $17 \%$ of respondents, a low cost technology, which would be a facilitator for its implementation in BHU, since it does not demand many resources to apply it.

I think it is totally possible to be applied in BHU because it does not depend on any more robust technology. (E6)

In the second moment, the perspective to continue using SNA continued to be seen with positive aspects by $75 \%$ of respondents, evidenced by the following report:

They are positive, I verified that it has to be used in our routine, initially with people with chronic diseases. (E1)

It was also evaluated as a form of organization by $100 \%$ of the nurses, reinforcing what was elucidated in the first stage of the research, demonstrating that it is possible to keep up with the organization of the service demands, in addition to the participants believing in the possibility of better treatment adherence as highlighted in $50 \%$ of the speeches, perspective maintained before and after the use of SNA:

I believe it is feasible to apply SNA in this way in hypertensive and diabetic consultations because it is a tool that is incorporated into the routine, it will have certain difficulties in the beginning, but over time it will give a practicality to identify the patient's demands. (E4)

If all the nurses of the BHU apply and register well in the medical record, even if the patient is consulted by another nurse he will get that continuity of care and not just repeat the same things, right? (E4)

These results corroborate with the study carried out by reference [12], with 20 nurses from two hospitals in which it resulted in categories describing SAE in: order, disorder and reorganization. It concludes that $\mathrm{SAE}$ is still seen with normative-legal issues, with an idea of order-to-service, and that it is capable of offering this integrity of care, further emphasizes that the disorder of its implementation is necessary, integral to the process, for the implantation systematization in the service.

The need for technologies was elucidated again in the second phase, where it is described as a means of improving the way in which SNA was implemented:

Any new technology, the improvement and maintanance of the conciseness, keeping lean, without removing the essential as is the case of the instrument, the rapid access to the diagnoses and prescriptions is fundamental to maintain the applicability in the future, a question to think would be the creation of a smartphone application, to put all this in an app where anyone can have access on the cell is going to be very good, extraordinary (E6).

In a research carried out in a University Hospital in Petrolina, Brazil, by reference [13], on the implementation of a nursing diagnosis and prescription application, had as good points practicality and agility, promoting material resource savings.

The perspectives in the evidenced statements of organization and improvement of care demonstrate the concern of nurses between them and their world of work, since systematizing care through the use of an instrument provides safety and agility in care when organizing the actions and guiding the nursing service. Therefore, the nurse should be willing to not perform it mechanically, reflecting on their actions, a targeted care, provides agility and objectivity in care [14].

\subsection{Difficulties Expected, Difficulties Found}

Among the difficulties detected in the interviewees' speech, prior to implantation, $83 \%$ considered the teaching of SNA practice insufficient in their graduation program, especially regarding their use in primary care, and that this makes it difficult to use the methodology in the environment.

I do not know if maybe due to lack of structure and organization of the places that we passed through internship, but the practical part, mainly at the end of the course we were not able to apply or associate the SNA in the health units that we went on. (E1)

In the reference study [15], it shows that investigated students also describe a difficulty between association of practice and theory, but they know the importance of SAE in care, where the authors reiterate the importance during graduation of direct contact with SAE and its phases of deployment.

It is up to the university and the faculty to work SNA, in order to prepare the future professionals of the area, although there are obstacles in the way like the reality of internship, absence of the use of SNA in some establishments, situations that hinder the didactic development of these professionals.

The lack of objectivity in the services was considered by $17 \%$ of the sample as a difficulty in implementing the SNA in the service.

And I realize that we need to be a bit more objective, we 
are the ones that are going to develop it, we are going to apply the SNA, maybe this objectivity is missing. (E3)

The work overload was observed in $33 \%$ of the interviewees as a negative point for SNA implementation, according to the interviewees, in the work routine there is no time to complete the five steps of the methodology.

Because sometimes in the routine we can't, we do not have much time to think about the diagnosis, right?, to get a diagnosis and to seek prescriptions for that diagnosis, right? (E4)

The difficulties of SAE implantation are present in several nursing scenarios, making it increasingly difficult to perform. In several intuitions there is no adherence and commitment of all to its use or implantation, the problems of its implantation go from theoretical-practical ignorance to difficulties that appear in the team [15].

Regarding teamwork, 33\% considered a difficulty in implementing the methodology in the environment, and 50\% associated this situation with the disinformation of the team and the professionals about the importance of the nursing consultation.

The main difficulty I think would be the lack of teamwork, a team more evenly joined, right? (E5)

Is that situation, right?, of the patient who arrives here and is already directed to medical consultation, how would you do that to bring firstly to the nursing consultation? (E5)

Although during nursing, or in courses and specializations are carried out on the SAE, or even if the nurse has legislation that guarantees as being a private service, which supports the profession with scientificity, nursing has difficulties for its adoption. However, when its correct use provides results that bring recognition of the medical team and results satisfactory to the patient, it generates in the nurse feelings of satisfaction and gratification, the SAE in its effectiveness, is the way of the nurse to have his work with quality [14].

The lack of computerization was also considered a difficulty in the applicability of the SNA in the UBH, since it makes it difficult to follow up the patient, regarding the attendance in the consultations and in the health service.

As we do not have computerized service, each and every person arrives and she has the prescription, she receives the medication and the service happens to be a dispenser of the medication. (E3)

In a literature review carried out by reference [16], among the results obtained about SNA, the use of software was placed as a facilitator of nursing actions, provided that all stages of the nursing process are attached to the electronic medical record.

After the implantation, it is observed that the only similar indicator between the two phases of the surveys is the overload of the mentioned service before the implantation and reinforced in the speeches of $75 \%$ of the interviewees, evidencing the necessity of organization of other demands of the service (other programs being serviced).

So it is also important to adapt our schedule because many times we receive many patients on the same day and consequently end up not having time to talk very well with each one. (E4).

The time was not the difficulty most evidenced in the first interview, however in the second moment it was evidenced in $100 \%$ of the speeches:

(...) length of service was a little bigger. (E1)

(...) the query extends slightly more. (E4)

(...) I think the difficulty is this, the patients I attended to were in a bit of a hurry, right?. (E5)

Among the highlights, the time and the overload of activities are pointed out in another study. The time when the service is organized can be optimized. The use of systematization, in the first instance, finds difficulties, such as prolonged care time, as well as other extrinsic factors such as lack of resources, arduous attribution scales, among others, can cause an initial disorder, rather than the practice of use, can minimize the difficulties encountered by the team [12-14].

In this sense, only experiencing the applicability of SNA in the institution will allow to observe the challenges and perspectives of its use. Thus, the difficulties evidenced before and after their use demonstrate the obstacles from the perspective of the interviewees, linked to an entire context associated with the evocation of their old and current experiences.

\subsection{Use of Sna in Primary Care: Before and After a Practical Experience}

In the interviewees' speeches at the first moment, the systematization of care was evidenced as a means of planning and organizing the nursing service, promoting objective and quality care, but in practice, only a few steps are performed satisfactorily.

The SNA is being regulated for nine years by the Federal Nursing Council by resolution n. 358/2009 that provides for its implementation in public or private environments that have the nursing service [1], it still identifies difficulties either in understanding or executing its stages, which makes non-systematized care unfeasible as evidenced in $100 \%$ of the speeches:

We end up practicing with certain acts, for example the planning part, the prescription part as isolated parts. They are not acts in a row. (E1)

Non-systematized care, without compliance with the five steps established in the methodology in question, decreases the effectiveness of care, since there are no evaluation parameters without the construction of diagnoses and prescriptions compatible with the problem detected during data collection, thus making it impossible to nursing care to be longitudinal.

Nowadays we practically do not use it, we do not have any way to systematize this care, these guidelines that we give to the patient we do in an empirical way, and in the best way that we think possible. (E4)

The use of the nursing process enables the provision of individualized care. It is a valuable instrument for nursing. It allows evaluation and documentation of the service provided, 
in which there is still a lack of knowledge in its implementation, in which there are a large number of establishments in the private and public network that does not perform the SAE, but this framework has been evolving, care requiring quality and improvement of nursing services [15-16].

To ensure the provision of quality customer service, the SNA should be implemented, as it is a process that has the tools for a good teamwork, but to implement it it is necessary to persist [17].

The practice of SNA should be part of the routine of every nurse, although initially in stages, since the lack of use of the method generates losses: for the patient who does not effectively adhere to the treatment, for the institution that does not have the structure to supply the needs of the client and for the nurse who does not demonstrate the relevance of systematized care and does not improve their technical knowledge [17].

In a reference study [10], there were numerous challenges for the implementation of SNA by nurses in the institutional routine where they work, among them: difficulties in implementing SNA correctly, lack of printouts, protocols, nursing shortages, which results in lack of time and the lack of training of professionals, so that this process ends up being fragmented to fit the reality of the institutions where these professionals are inserted.

Although the use of SNA in the service has required a longer time and this to be pointed out as the main difficulty, after the application of SNA, this same time was described as not being an obstacle to SNA execution, it was seen by $50 \%$ as a time that ensures better quality consultation:

But I did not verify that this was hindering the consultation, the patient herself and the companion they even talked about the duration of the consultation, but even as something positive, they referred as a complete consultation. (E1)

The SAE guides the care practices, the nurse must understand that it is a method that offers benefits to the profession [12]. When nursing empowers itself, and uses systematization as the scientific source of its action plan, it will consequently reflect in its assistance, and it is through positive results that it will demonstrate its importance to the user of the service.

The use of SNA was seen as a methodology that improves attendance, as well as a possibility to apply the theoretical part that in the first moment all reported to have.

Regarding APS, the use of SAE also presents difficulties, as well as in other fields of nursing practice, where SAE occurs in an incipient way, a behavior that can be attributed to training, where the use of systematization usually occurs predominantly in hospital settings. The use of SAE in APS requires greater institutional engagement through training and protocols to improve and facilitate the effectiveness of implementation of this assistance model [18].

The use of SNA in the PHC field in the care of chronically ill patients made possible to perceive that its use brings improvements to the consultation, although in principle it has an impact on the nurses' work routine, since it requires time and skill to use it, but in long-term, it will organize the service and improve the quality of patient care, as evidenced in the speeches of the participants after their implementation.

\section{Conclusion}

Although the improvement in the quality of the care and greater effectiveness in the nursing actions regarding the application of SNA to the chronic patient is undeniable, some obstacles still stand in relation to the implementation of the method. The SNA implementation presents difficulties in the beggining as evidenced in the results of this study, however, in the medium term it will bring more benefits than any identified burden for the assistance and management of human resources and materials of the health unit.

\section{References}

[1] Brasil, Conselho Federal de Enfermagem. Resolução cofen358/2009.

[2] Da Silva CG, Sena LB, Rolim ILTP, De Souza SMA, Sardinha AHLS. Cuidados de enfermagem a pacientes com condições crônicas de saúde: uma revisão integrativa. Revista de Pesquisa: Cuidado é Fundamental Online, [S. 1], 2017; 9 (2): 599-605.

[3] Mendes EV. O cuidado das condições crônicas na atenção primária à saúde. Rev Bras Promoç Saúde, Fortaleza, abr/jun, 2018; 31 (2): 1-3.

[4] Sociedade Brasileira de Cardiologia. $7^{\text {a }}$ diretriz brasileira de hipertensão arterial. Arquivos Brasileiros de Cardiologia, Rio de Janeiro, 2016; 107 (3).

[5] Sociedade Brasileira de Diabetes. Diretrizes SBD 2017-2018. São Paulo: AC Farmacêutica, 2017.

[6] Becker RM, Heidemann ITSB, Meirelles BHS, Da Costa MFBNA, Antonini FO, Durand MK. Práticas de cuidado dos enfermeiros a pessoas com Doenças Crônicas Não Transmissíveis. Rev Bras Enferm [Internet]. 2018;71 (suppl 6): 2800-7.

[7] Brasil. Ministério da saúde. Portaria No 2.436/2017.

[8] Cavalcante RB, Calixto P, Pinheiro MMK. Análise de Conteúdos: Considerações gerais, relações com a pergunta de pesquisa, possibilidades e limitações do método. Inf\&Soc.: Est., João Pessoa, 2014; 22 (1): 13-18.

[9] Bardin L. Análise de Conteúdo. 70 ed. São Paulo. 2011. P. 48.

[10] Soares MI, Resck, ZMR, Terra FS, Camelo SHH. Sistematização da assistência de enfermagem: facilidades e desafios do enfermeiro na gerência da assistência. Esc Anna Nery, 2015; 19 (1): 47-53.

[11] Gandolfi M, Siega CK, Rostirolla LM, Kleba ME, Colliselli L. Sistematização da assistência de enfermagem: da teoria ao cuidado integral. Rev enferm UFPE on line., Recife, set., 2016; 10 (Supl. 4): 3694-703.

[12] Dotto, JI, Backes DS, Dalcin CB, Filho WDL, De Siqueira $\mathrm{HCH}$, Zamberlan C. Sistematização da assistência de enfermagem: ordem, desordem ou (re) organização?. Rev enferm UFPE on line., Recife, out., 2017; 11 (10): 3821-9. 
[13] Alencar IGM, Nunes VS, Alves AS, Cruz RPG. Estratégia de implantação de aplicativo para prescrição de enfermagem. Rev Enferm UFPE On line, Recife, 2018; 12 (1): 273-9.

[14] Gonçalves MRCB, Spiri WC, Ortolan EVP. Sentimento dos enfermeiros de um hospital universitário quanto à prática diária do processo de enfermagem. Rev enferm UFPE on line., Recife, Maio, 2017; 11 (5): 1902-8.

[15] Da Silveira V, Da Silva KC, Hertel VL. Sistematização da assistência de enfermagem na saúde da família: percepção dos acadêmicos de enfermagem. Rev enferm UFPE on line. Recife, nov., 2016; 10 (11): 3892-900.
[16] Campos NPS, Rosa CA, Gonzaga MFN. Dificuldades na implementação da sistematização de enfermagem. Rev Saúde em Foco. 2017; 9 ed.

[17] Barbosa EP, De Biasi LS, Zago VLS, Paini JP, Severo CM. Sistematização da assistência de Enfermagem: Dificuldades de implantação na visão do enfermeiro. PERSPECTIVA, Erechim, 2012; 36 (133): 41-51.

[18] Ribeiro GC, Padoveze MC. Sistematização da Assistência de Enfermagem em unidade básica de saúde: percepção da equipe de enfermagem* Rev Esc Enferm USP, 2018; 52: $\mathrm{e} 03375$. 